\title{
On Gravitational anomaly and Hawking radiation near weakly isolated horizon
}

\author{
Xiaoning $\mathrm{Wu}^{a, c *}$, Chao-Guang Huang ${ }^{b, c \dagger}$, and Jia-Rui Sun ${ }^{b, d \ddagger}$ \\ ${ }^{a}$ Institute of Mathematics, Academy of Mathematics and Systems Science, \\ Chinese Academy of Sciences, P.O.Box 2734, Beijing, 100080, China \\ ${ }^{b}$ Institute of High Energy Physics, Chinese Academy of Sciences, \\ P.O. Box 918(4), Beijing, 100049, China
}

${ }^{c}$ Kavli Institute for Theoretical Physics China at the Chinese Academy of Sciences (KITPC-CAS), P.O.Box 2732, Beijing, 100080, China and

${ }^{d}$ Graduate School of Chinese Academy of Sciences, Beijing, 100049, China.

Based on the idea of the work by Wilczek and his collaborators, we consider the gravitational anomaly near weekly isolated horizon. We find that there exists a universal choice of tortoise coordinate for any weakly isolated horizon. Under this coordinate, the leading behavior of a quite arbitrary scalar field near horizon is a 2-dimensional chiral scalar field. This means we can extend the idea of Wilczek and his collaborators to more general cases and show the relation between gravitational anomaly and Hawking radiation is a universal property of black hole horizon.

PACS numbers: 04.70.Dy

\footnotetext{
* Email: wuxn@amss.ac.cn

$\dagger$ Email: huangcg@ihep.ac.cn

¥ Email: sun@ihep.ac.cn
} 


\section{INTRODUCTION}

Hawking radiation and black hole thermal dynamics is believed to be most important evidence for the deep relation between quantum theory and general relativity. After Hawking's original paper [1, 2], many works have been done in this area in order to get deeper understanding on the properties of quantum fields in curved spacetimes. Many methods have been developed in last several decades. They all show that the phenomena of Hawking radiation exists for many kinds of black hole [3]-[10], including for some non-stationary black holes [11]-[13]. Recently, Wilczek and his collaborators proposed a new method based on the anomaly analysis [14, 15]. The anomaly analysis in the study of the Hawking radiation can be traced back to Christensen and Fulling [16]. They consider the trace anomaly of a conformally invariant scalar field in Schwarzschild background and show that there is a relation between the Hawking radiation and anomalous trace of the field under the condition that the covariant conservation law is valid. By imposing a boundary condition near horizon, Wilczek and his collaborators prove that the Hawking radiation is just the cancel term of the gravitational anomaly of the covariant conservation law [14] and gauge invariance as well [15]. Later, this idea is extended to other kinds of black holes[17]-[27]. The aim of this paper is to generalize the approach of Wilczek and his collaborators to more general cases, including dynamical black holes. To do so, a general definition of a horizon is needed since the event horizon of black hole cannot describe the dynamical properties of black hole very well [28]. The weakly isolated horizon is the appropriate notation to replace the event horizon. Thus, we shall show in this paper that the approach is available to the weakly isolated horizons.

The organization of this paper is as follows. In section II, we briefly review the definition of weakly isolated horizon and the geometry near it in the Bondi-like coordinate system with Bondi-gauge. Some detail analysis on the asymptotic behavior of the d'Alembert operator near horizon is also made in this section. Section III gives the calculation of gravitational anomaly near a weakly isolated horizon. Section IV focuses on the gauge anomaly of electromagnetic field. In section $\mathrm{V}$, the analysis is generalized to weakly isolated horizons in higher dimensional spacetimes. Section VI contains some discussions.

\section{PRELIMINARIES}

During last decade, motivated by the need of numerical relativity and relativistic astrophysics, much work has been done to find a quasi-local definition of a black hole. A very nice review on the development on the quasi-local definition of a horizon and its possible applications can be found in Ref.[28]. In the present paper, we will follow the definition of the weakly isolated horizon, given by Ashtekar and his collaborators.

Definition 1 (Weakly Isolated Horizon)

Let $(M, g)$ be a space-time. $\mathcal{H}$ is a 3-dim null hyper-surface in $M$ and $l^{a}$ is the tangent vector field of the generator of $\mathcal{H} . \mathcal{H}$ is said to be a weakly isolated horizon(WIH), if

1) $\mathcal{H}$ has the topology of $S^{2} \times \mathbf{R}$;

2) The expansion of the null generator of $\mathcal{H}$ is zero, i.e. $\Theta_{l}=0$ on $\mathcal{H}$;

3) $T_{a b} v^{b}$ is future causal for any future causal vector $v^{a}$ and Einstein equation holds in a neighborhood of $\mathcal{H}$;

4) $\left[\mathcal{L}_{l}, D_{a}\right] l^{b}=0$ on $\mathcal{H}$, where $D_{a}$ is the induced covariant derivative on $\mathcal{H}$. 
By definition, it can be shown that there exists a 1 -form $\omega_{a}$ on $\mathcal{H}$ such that $D_{a} l^{b} \hat{=} \omega_{a} l^{b}$, where $\hat{=}$ denotes the equality restricted to $\mathcal{H}$. Similarly, for quantity $f$ on spacetime, $\hat{f}$ means the value of $f$ on horizon $\mathcal{H}$, following the notation in Ref. [28].

As in Ref. 29], we can introduce a Bondi-like coordinates $(u, r, \theta, \varphi)$ in a neighborhood of horizon $\mathcal{H}$ in the following way. First, denote the tangent vector of null generator of $\mathcal{H}$ as $l^{a}$ and another real null vector field as $n^{a}$. Then, the foliation of $\mathcal{H}$ gives us the natural coordinates $(\theta, \varphi)$. Their Lie drag along each generator of $\mathcal{H}$ together with the parameter $u$ of $l^{a}$ form the coordinates on $\mathcal{H}$. Finally, choose the affine parameter $r$ of $n^{a}$ as the forth coordinate. Furthermore, we can also choose a set of null tetrad which satisfy Bondi gauge in this neighborhood [30, 31]. The expression of the tetrad in Bondi coordinates are

$$
\left\{\begin{array}{l}
l^{a}=\partial_{u}+U \partial_{r}+X \partial_{\zeta}+\bar{X} \partial_{\bar{\zeta}} \\
n^{a}=\partial_{r} \\
m^{a}=\omega \partial_{r}+\xi^{3} \partial_{\zeta}+\xi^{4} \partial_{\bar{\zeta}} \\
\bar{m}^{a}=\bar{\omega} \partial_{r}+\bar{\xi}^{4} \partial_{\zeta}+\bar{\xi}^{3} \partial_{\bar{\zeta}}
\end{array}\right.
$$

where

$$
U \hat{=} X \hat{=} \omega \hat{=} 0
$$

and $\zeta=e^{i \varphi} \cot \frac{\theta}{2}$. Then the metric takes the form [31]

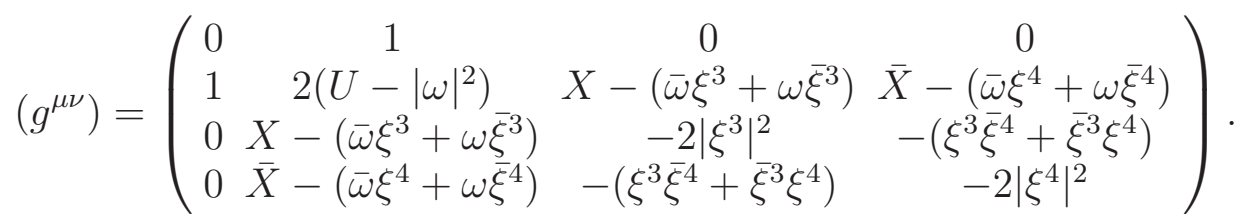

It is easy to see that $\sqrt{-g}=\sqrt{h}$ if we denote $\left(h_{A B}\right):=-\left(\xi^{A} \bar{\xi}^{B}+\bar{\xi}^{A} \xi^{B}\right)^{-1}$ with $A, B=3,4$. Obviously, $h_{A B}$ is the induced metric on the section of WIH.

In the Newman-Penrose formalism, the Bondi gauge can be expressed as

$$
\nu=\tau=\gamma=\alpha+\bar{\beta}-\pi=\mu-\bar{\mu}=0, \quad \varepsilon-\bar{\varepsilon} \hat{=} \kappa \hat{=} 0,
$$

where

$$
\begin{aligned}
-\nu & =n_{\mu ; \nu} \bar{m}^{\mu} n^{\nu}=\bar{m}^{\mu} \Delta n_{\mu}, \\
\tau & =l_{\mu ; \nu} m^{\mu} n^{\nu}=m^{\mu} \Delta l_{\mu}, \\
-\pi & =n_{\mu ; \nu} \bar{m}^{\mu} l^{\nu}=\bar{m}^{\mu} D n_{\mu}, \\
-\mu & =n_{\mu ; \nu} \bar{m}^{\mu} m^{\nu}=\bar{m}^{\mu} \delta n_{\mu}, \\
\kappa & =l_{\mu ; \nu} m^{\mu} l^{\nu}=m^{\mu} D l_{\mu}, \\
-\gamma & =\frac{1}{2}\left(n_{\mu ; \nu} l^{\mu} n^{\nu}-\bar{m}_{\mu ; \nu} m^{\mu} n^{\nu}\right)=\frac{1}{2}\left(l^{\mu} \Delta n_{\mu}-m^{\mu} \Delta \bar{m}_{\mu}\right), \\
-\alpha & =\frac{1}{2}\left(n_{\mu ; \nu} l^{\mu} \bar{m}^{\nu}-\bar{m}_{\mu ; \nu} m^{\mu} \bar{m}^{\nu}\right)=\frac{1}{2}\left(l^{\mu} \bar{\delta} n_{\mu}-m^{\mu} \bar{\delta} \bar{m}_{\mu}\right), \\
\beta & =\frac{1}{2}\left(l_{\mu ; \nu} n^{\mu} m^{\nu}-m_{\mu ; \nu} \bar{m}^{\mu} m^{\nu}\right)=\frac{1}{2}\left(n^{\mu} \delta l_{\mu}-\bar{m}^{\mu} \delta m_{\mu}\right), \\
\varepsilon & =\frac{1}{2}\left(l_{\mu ; \nu} n^{\mu} l^{\nu}-m_{\mu ; \nu} \bar{m}^{\mu} l^{\nu}\right)=\frac{1}{2}\left(n^{\mu} D l_{\mu}-\bar{m}^{\mu} D m_{\mu}\right),
\end{aligned}
$$


where $D:=l^{a} \nabla_{a}, \Delta:=n^{a} \nabla_{a}, \delta:=m^{a} \nabla_{a}$ and $\bar{\delta}:=\bar{m}^{a} \nabla_{a}$ as the standard notation in [30]. Other three spin coefficients are

$$
\begin{aligned}
\rho & =l_{\mu ; \nu} m^{\mu} \bar{m}^{\nu}=m^{\mu} \bar{\delta} l_{\mu}, \\
\sigma & =l_{\mu ; \nu} m^{\mu} m^{\nu}=m^{\mu} \delta l_{\mu}, \\
-\lambda & =n_{\mu ; \nu} \bar{m}^{\mu} \bar{m}^{\nu}=\bar{m}^{\mu} \bar{\delta} n_{\mu} .
\end{aligned}
$$

In the Bondi gauge, 1 -form $\omega_{a}$ is expressed as

$$
\omega_{a}=-(\varepsilon+\bar{\varepsilon}) n_{a}+(\alpha+\bar{\beta}) \bar{m}_{a}+(\bar{\alpha}+\beta) m_{a}=-(\varepsilon+\bar{\varepsilon}) n_{a}+\pi \bar{m}_{a}+\bar{\pi} m_{a}
$$

and $\left.(\varepsilon+\bar{\varepsilon})\right|_{\mathcal{H}}$ is constant [28]. In addition, the definition of WIH implies

$$
\rho \hat{=} \sigma \hat{=} 0
$$

Based on the result in Ref. [28], the angular momentum of WIH is

$$
J[\varphi]=-\frac{1}{8 \pi} \int_{S}\left(\varphi^{a} \omega_{a}\right) d V_{2},
$$

where $\varphi^{a}$ is a vector field on section $S$. So, among the NP coefficients, only $\pi$ is related to the angular momentum of WIH.

The commutators of the null tetrad require

$$
\begin{aligned}
& U=(\hat{\varepsilon}+\hat{\bar{\varepsilon}}) r+O\left(r^{2}\right), \kappa=O(r), \alpha=O(1), \mu=O(1), \\
& \varepsilon=\hat{\varepsilon}+O(r), \quad \frac{\partial \xi^{3}}{\partial u} \hat{=} 0, \quad \beta=O(1), \lambda=O(1), \\
& \pi=O(1), \quad \frac{\partial \xi^{4}}{\partial u} \hat{=} 0, \quad \omega=O(r) .
\end{aligned}
$$

The d'Alembert operator can be re-written as

$$
\begin{aligned}
\square= & \left(l^{a} n^{b}+n^{a} l^{b}-m^{a} \bar{m}^{b}-\bar{m}^{a} m^{b}\right) \nabla_{a} \nabla_{b} \\
= & D \Delta+\Delta D+(\mu+\bar{\mu}) D+(\varepsilon+\bar{\varepsilon}) \Delta-(\rho+\bar{\rho}) \Delta-\pi \delta-\bar{\pi} \bar{\delta}-\Delta_{S} \\
= & 2 \partial_{u} \partial_{r}+2 U\left(\partial_{r}\right)^{2}+2 X \partial_{r} \partial_{\zeta}+2 \bar{X} \partial_{r} \partial_{\zeta} \\
& +\frac{\partial U}{\partial r} \partial_{r}+\frac{\partial X}{\partial r} \partial_{\zeta}+\frac{\partial \bar{X}}{\partial r} \partial_{\bar{\zeta}}+(\mu+\bar{\mu})\left(\partial_{u}+U \partial_{r}+X \partial_{\zeta}+\bar{X} \partial_{\bar{\zeta}}\right) \\
& +(\varepsilon+\bar{\varepsilon}) \partial_{r}-(\rho+\bar{\rho}) \partial_{r}-\pi \delta-\bar{\pi} \bar{\delta}-\delta \bar{\delta}-\bar{\delta} \delta+(\alpha-\bar{\beta}) \delta+(\bar{\alpha}-\beta) \bar{\delta}
\end{aligned}
$$

Detail calculation also tells us

$$
\Delta_{S}=\delta \bar{\delta}+\bar{\delta} \delta-(\alpha-\bar{\beta}) \delta-(\bar{\alpha}-\beta) \bar{\delta}+O\left(r^{2}\right)
$$

where $\Delta_{S}$ is the Laplacian on the coordinate 2-sphere.

\section{GRAVITATIONAL ANOMALY}

In this section, we will show that the relation between Hawking radiation and gravitational anomaly still exists for weakly isolated horizons. 
Let's consider a scalar field near a weakly isolated horizon, whose action can be written as

$$
S=-\frac{1}{2} \int d V_{4} \phi\left(\square-m^{2}\right) \phi .
$$

Using Eq.(7), it can be written in the explicit form in Bondi-like coordinate system

$$
\begin{aligned}
S= & -\frac{1}{2} \int d u d r d \theta d \varphi \sqrt{h} \phi\left[2 \partial_{u} \partial_{r}+2 U\left(\partial_{r}\right)^{2}+2 X \partial_{r} \partial_{\zeta}+2 \bar{X} \partial_{r} \partial_{\zeta}\right. \\
& +\left(\partial_{r} U\right) \partial_{r}+\left(\partial_{r} X\right) \partial_{\zeta}+\left(\partial_{r} \bar{X}\right) \partial_{\bar{\zeta}}+(\mu+\bar{\mu})\left(\partial_{u}+U \partial_{r}+X \partial_{\zeta}+\bar{X} \partial_{\bar{\zeta}}\right) \\
& +(\varepsilon+\bar{\varepsilon}) \partial_{r}-(\rho+\bar{\rho}) \partial_{r}-\pi\left(\omega \partial_{r}+\xi^{3} \partial_{\zeta}+\xi^{4} \partial_{\bar{\zeta}}\right) \\
& \left.-\bar{\pi}\left(\bar{\omega} \partial_{r}+\bar{\xi}^{4} \partial_{\zeta}+\bar{\xi}^{3} \partial_{\bar{\zeta}}\right)-\Delta_{S}+O\left(r^{2}\right)-m^{2}\right] \phi
\end{aligned}
$$

Introduce new coordinates in a neighborhood of WIH as

$$
t:=u-r_{*}, \quad R:=r
$$

where $d r_{*}=d r / f(r)$ and $f(r)=2(\hat{\varepsilon}+\hat{\bar{\varepsilon}}) r+O\left(r^{2}\right)$. Then it is easy to get

$$
\partial_{u}=\partial_{t}, \quad \partial_{r}=-f^{-1} \partial_{t}+\partial_{R}
$$

Denote $\left\{\lambda_{k}\right\}$ are eigenvalues of the Laplacian $\Delta_{S}$ and $\left\{F_{k}\right\}$ are associated (normalized) eigenfunctions, and make the variable separation for the scalar field $\phi$ as

$$
\phi(u, r, \theta, \varphi)=\sum_{k} \phi_{k}(u, r) F_{k}(\theta, \varphi) .
$$

Then, the action becomes

$$
\begin{aligned}
S= & -\frac{1}{2} \sum_{k} \int d t d R \cdot \phi_{k}\left[-\frac{1+O(r)}{f}\left(\partial_{t}\right)^{2}+[1+O(r)] \partial_{R}\left(f \partial_{R}\right)+O(r) \partial_{t} \partial_{R}\right. \\
& +\frac{O(r)}{f} \partial_{t}+\frac{1}{2}[\mu+\bar{\mu}+O(r)] \partial_{t}+(\mu+\bar{\mu})\left[(\hat{\varepsilon}+\hat{\bar{\varepsilon}}) r+O\left(r^{2}\right)\right] \partial_{R} \\
& \left.+\frac{\rho+\bar{\rho}}{f} \partial_{t}-(\rho+\bar{\rho}) \partial_{R}+\frac{\pi \omega+\bar{\pi} \bar{\omega}}{f} \partial_{t}-(\pi \omega+\bar{\pi} \bar{\omega}) \partial_{R}-\lambda_{k}+\tilde{O}(r)\right] \phi_{k} \\
& -\frac{1}{2} \sum_{k, k^{\prime}} \int d t d R f^{-1} \phi_{k}(t, R) O_{k k^{\prime}}(t, R) \phi_{k^{\prime}}(t, R) .
\end{aligned}
$$

Here the symbol $\tilde{O}(r)$ represents an $O(r)$ operator without $O(r) \partial_{r}$ terms and

$$
\begin{aligned}
O_{k k^{\prime}}(t, R)= & \int d \theta d \varphi \sqrt{h} F_{k}(\theta, \varphi)\left[2 X\left(-\partial_{t}+f \partial_{R}\right) \partial_{\zeta}+2 \bar{X}\left(-\partial_{t}+f \partial_{R}\right) \partial_{\zeta}\right. \\
& +\left(-\partial_{t} X+f \partial_{R} X\right) \partial_{\zeta}+\left(-\partial_{t} \bar{X}+f \partial_{R} \bar{X}\right) \partial_{\bar{\zeta}}+(\mu+\bar{\mu}) f\left(X \partial_{\zeta}+\bar{X} \partial_{\bar{\zeta}}\right) \\
& \left.-\pi f\left(\xi^{3} \partial_{\zeta}+\xi^{4} \partial_{\bar{\zeta}}\right)-\bar{\pi} f\left(\bar{\xi}^{4} \partial_{\zeta}+\bar{\xi}^{3} \partial_{\bar{\zeta}}\right)\right] F_{k^{\prime}}(\theta, \varphi)
\end{aligned}
$$

is another $\tilde{O}(r)$ operator. In the tortoise coordinate $d R_{*}=d R / f$, the action reduces to

$$
\begin{aligned}
S= & -\frac{1}{2} \sum_{k} \int d t d R_{*} \phi_{k}\left[-\left(\partial_{t}\right)^{2}+\left(\partial_{R_{*}}\right)^{2}+O(r)\right] \phi_{k} \\
& -\frac{1}{2} \sum_{k, k^{\prime}} \int d t d R_{*} \phi_{k}(t, R) O_{k k^{\prime}}(t, R) \phi_{k^{\prime}}(t, R) .
\end{aligned}
$$


With respect to coordinate $R_{*}$, the term $O(r)$ vanishes exponentially, so the dominant term of the action (8) near a weakly isolated horizon takes the form of an infinite collection of 2-dimensional fields. The metric of the effective 2-dimensional spacetime is

$$
d s^{2}=-f(R) d t^{2}+\frac{d R^{2}}{f(R)},
$$

and the horizon is the boundary of the effective spacetime. This behavior is similar to what happens near Schwarzschild black hole horizon [14].

As in Ref. [14, 17], the effective 2-dimensional spacetime is bounded by the horizon on one side (at $R=r=0$ ), on which the boundary condition that the outgoing modes vanish is imposed. Then, in the "near horizon region", $0<r<a$ with $a \rightarrow 0$, the fields become chiral. It is well-known that a $(4 k+2)$-dimensional chiral theory contains the following gravitational anomaly [32] 35$]$

$$
\nabla_{\mu} T_{\nu}^{\mu}=\frac{1}{96 \pi \sqrt{-g}} \epsilon^{\beta \alpha} \partial_{\alpha} \partial_{\eta} \Gamma_{\nu \beta}^{\eta}
$$

where $\alpha, \beta, \eta, \mu, \nu=0,1, g$ is the determinant of $(4 k+2)$-dimensional metric, and the convention $\epsilon^{01}=1$ is used. The divergence of the energy-momentum tensor can be generally written as

$$
\nabla_{\mu} T_{\nu}^{\mu}=\mathscr{A}_{\nu}=\frac{1}{\sqrt{-g}} \partial_{\mu} N_{\nu}^{\mu} .
$$

In the "out region", $r>a$, there is no anomaly in the divergence of the energy-momentum tensor. Therefore, $N_{\nu}^{\mu}=0$ and thus $\mathscr{A}_{\nu}=0$. In near horizon region, from the metric (16) and Eq.(17), it is easy to see

$$
N_{t}^{t}=N_{R}^{R}=0, \quad N_{t}^{R}=\frac{1}{192 \pi}\left(f f^{\prime}\right)^{\prime}, \quad N_{R}^{t}=\frac{1}{192 \pi}\left(f^{-1} f^{\prime}\right)^{\prime},
$$

where a prime means $\partial_{R}$ and thus

$$
\mathscr{A}_{t}=\frac{1}{192 \pi}\left(f f^{\prime}\right)^{\prime \prime}, \quad \mathscr{A}_{R}=0
$$

The effective action for the metric after integrating out the field $\phi$ is

$$
W\left[g_{\mu \nu}\right]=-i \ln \left(\int \mathcal{D}[\phi] \exp \left(i S\left[\phi, g_{\mu \nu}\right]\right)\right),
$$

where $S\left[\phi, g_{\mu \nu}\right]$ is the classical action. A basic requirement for a well-defined quantum theory is that it should be anomaly free. In the present case, it is equivalent to require that the full quantum theory is diffeomorphism invariant. The requirement can be expressed in terms of $W\left[g_{\mu \nu}\right]$. Suppose $v^{a}$ to be a vector field, $\delta_{v}$ is the variation induced by $v^{a}$. Under the variation induced by any $v^{a} \in T M$,

$$
\begin{aligned}
-\delta_{v} W= & \int d^{2} x \sqrt{-g} v^{\nu} \nabla_{\mu}\left[T_{\chi}^{\mu} \Theta_{-}+T_{O \nu}^{\mu} \Theta_{+}\right] \\
= & \int d^{2} x v^{t}\left[\partial_{R}\left(N_{t}^{R} \Theta_{-}\right)+\left(T_{O t}^{R}-T_{\chi}^{R}+N_{t}^{R}\right) \partial_{R} \Theta_{+}\right] \\
& +\int d^{2} x v^{R}\left(T_{O R}^{R}-T_{\chi R}^{R}\right) \partial_{R} \Theta_{+} \cdot
\end{aligned}
$$


where $T_{\chi}{ }_{\nu}{ }_{\nu}$ and $T_{O}{ }_{\nu}{ }_{\nu}$ are the energy-momentum tensor in near horizon and in the out region, respectively, as the notation in [14], and $\Theta_{+}=\Theta(r-a)$ and $\Theta_{-}=1-\Theta_{+}$are step functions.

To obtain the concrete expression for the variation of effective action, the explicit expression for the energy-momentum tensor is needed. Because the effective metric is static, $T_{\nu}^{\mu}$ is independent of $t$. Then the general solutions of Eq.(18) for $T_{\chi}{ }_{\nu}{ }_{\nu}$ and $T_{O}{ }_{\nu}$ are

$$
\left\{\begin{array}{l}
T_{t}^{t}=-\frac{K+Q}{f}-\frac{B(R)}{f}-\frac{I(R)}{f}+T(R), \\
T_{R}^{R}=\frac{K+Q}{f}+\frac{B(R)}{f}+\frac{I(R)}{f} \\
T_{t}^{R}=-K+C(R)=-f^{2} T_{R}^{t},
\end{array}\right.
$$

where

$$
\left\{\begin{array}{l}
C(R)=\int_{0}^{R} \mathscr{A}_{t}(s) d s \\
B(R)=\int_{0}^{R} f(s) \mathscr{A}_{R}(s) d s \\
I(R)=\frac{1}{2} \int_{0}^{R} T(s) f^{\prime}(s) d s
\end{array}\right.
$$

and $T(R)$ is the trace of energy-momentum tensor. Therefore, the variation of the effective action becomes

$$
\begin{gathered}
-\delta_{v} W=\int d^{2} x v^{t}\left[\partial_{R}\left(N_{t}^{R} \Theta_{-}\right)+\left(N_{t}^{R}+K_{\chi}-K_{O}\right) \delta(R-a)\right] \\
+\int d^{2} x v^{R} \frac{K_{O}+Q_{O}-K_{\chi}-Q_{\chi}}{f} \delta(R-a) .
\end{gathered}
$$

The requirement that $\lim _{a \rightarrow 0} \delta_{v} W=0$ for any vector field $v^{a}$ demands

$$
\begin{aligned}
& K_{O}=K_{\chi}+\Phi \\
& Q_{O}=Q_{\chi}-\Phi \\
& \Phi=N_{t}^{R}(0)=\frac{1}{48 \pi}(\hat{\varepsilon}+\hat{\bar{\varepsilon}})^{2} .
\end{aligned}
$$

On the other hand, the surface gravity of a WIH is $\kappa_{l}=l^{a} \omega_{a}=(\hat{\varepsilon}+\hat{\bar{\varepsilon}})[28]$. So,

$$
\Phi=\frac{\kappa_{l}^{2}}{48 \pi}
$$

This means the gravitational anomaly near WIH has similar behavior as in Schwarzschild spacetime [14].

The total energy-momentum tensor $T_{\nu}^{\mu}=T_{\chi \nu}^{\mu}+T_{O \nu}^{\mu}$ can be rewritten, in the limit $a \rightarrow 0$, in two parts:

$$
T_{\nu}^{\mu}=T_{c \nu}^{\mu}+T_{\Phi \nu}^{\mu}
$$

where $T_{c \nu}^{\mu}$ is the conserved energy-momentum tensor of matter field which behaves as without any quantum effects, and $T_{\Phi \nu}^{\mu}$ is a conserved tensor with $K=-Q=\Phi$, a pure flux, which 
appears as the requirement to cancel the gravitational anomaly. Since Eq.(28) has the same form as the flux of black body radiation in $R$ direction at temperature $T$ in $2 \mathrm{D}$ spacetime, it is just a thermal radiation with the Hawking temperature $T=\kappa_{l} /(2 \pi)$.

A remark on the relation between our results and the Planck distribution is needed. It is well-known that a quantum field theory in curved space-time deeply depends on the choice of the observer. Unruh effect is a quite nice example. In this section, the observers which we used are rest ones in the "rest frame" in terminology of Ashtekar et al [28]. Concretely, the time direction is $\partial_{t}$ and the coordinate system is $(t, R, \theta, \varphi)$. In the coordinate system, a mode state of scaler field $\phi(x)$ labeled by quantum number $E$ and $m$ is

$$
\phi \propto \exp (-i E t+i m \varphi)
$$

and the distribution function observed by the observer, following the argument in Ref. [14, 15, 18], is $(\exp (E / T)+1)^{-1}$.

However, as emphasized in Ref.[28], not every choice of time direction will result in a Hamiltonian evolution in the phase space, then a horizon mass and first law of black hole thermal dynamics. In order to obtain the black hole mechanical law, one has to choose the canonical time. In non-rotational cases, the difference between our $t$ and the canonical one is higher order terms so it makes no contribution. In contrast, the non-zero horizon angular momentum will change all things. In the latter case, the leading term of the canonical time is

$$
\partial_{t_{c}} \hat{=} \partial_{t}+\Omega_{t} \partial_{\varphi}
$$

where $\partial_{\varphi}$ is the Killing vector for the metric on the 2-dimensional section of horizon and $\Omega_{t}$ is the angular velocity of the horizon. What is interested in is the radiation seen by the canonical observers. In the coordinates of a canonical observer near the horizon, $\left(t_{c}, R_{c}, \theta_{c}, \varphi_{c}\right)$, which is defined by

$$
t_{c}=t, R_{c}=R, \theta_{c}=\theta, \varphi_{c}=\varphi-\Omega_{t} t
$$

the mode state should be

$$
\phi \propto \exp \left[-i\left(E-m \Omega_{t}\right) t_{c}+i m \varphi_{c}\right]
$$

Then the distribution function observed by a canonical observer should be

$$
\frac{1}{\exp \left(\frac{E-m \Omega_{t}}{T}\right)+1} .
$$

This is the Planck spectrum with non-zero chemical potential. It means that a rotational isolated horizon has the same radiation spectrum as Kerr black hole [17, 18].

\section{GAUGE ANOMALY}

Now, let us turn to consider the gauge anomaly near WIH. The action of a complex scalar field $\phi(x)$ near WIH coupled to electromagnetic field is

$$
\begin{aligned}
S & =\frac{1}{2} \int d V_{4}\left\{\left[\left(\nabla_{a}-i e A_{a}\right) \bar{\phi}\right] \cdot\left[\left(\nabla^{a}+i e A^{a}\right) \phi\right]+m^{2}|\phi|^{2}\right\} \\
& =\frac{1}{2} \int d V_{4}\left\{\bar{\phi}\left[-\square+m^{2}\right] \phi-i e \bar{\phi} A_{a} \nabla^{a} \phi+i e \phi A_{a} \nabla^{a} \bar{\phi}+e^{2} \bar{\phi}|A|^{2} \phi\right\} .
\end{aligned}
$$


In the second equality a surface term is omitted.

The first interaction term in the integrand reads in the coordinate systems used in the previous sections

$$
\begin{aligned}
-i e \bar{\phi} A_{a} \nabla^{a} \phi= & -i e \bar{\phi}\left[f^{-1}\left(-A_{u}+f A_{r}-\mathcal{A} A_{r}-A_{A} \mathcal{B}^{A}\right) \partial_{t}\right. \\
& \left.\quad+\left(A_{u}+\mathcal{A} A_{r}+A_{A} \mathcal{B}^{A}\right) \partial_{R}+\left(A_{r} \mathcal{B}^{A}+h^{A B} A_{B}\right) \partial_{A}\right] \phi \\
= & -i e f^{-1} \bar{\phi}\left[-A_{u} \partial_{t}+\left(A_{u}+\mathcal{A} A_{r}+A_{A} \mathcal{B}^{A}\right) \partial_{R_{*}}+\tilde{O}(r)\right] \phi
\end{aligned}
$$

where $\mathcal{A}=2\left(U-|\omega|^{2}\right), \mathcal{B}^{3}=X-\left(\bar{\omega} \xi^{3}+\omega \bar{\xi}^{3}\right), \mathcal{B}^{4}=\bar{X}-\left(\omega \bar{\xi}^{3}+\bar{\omega} \xi^{3}\right), \partial_{3}=\partial_{\zeta}, \partial_{4}=\partial_{\bar{\zeta}}$. Under the coordinate transformation (11),

$$
A_{t}=A_{u}, A_{R}=f^{-1} A_{u}+A_{r}, A_{\zeta}=A_{\zeta} \text { and } A_{\bar{\zeta}}=A_{\bar{\zeta}}
$$

Thus,

$$
-i e \bar{\phi} A_{a} \nabla^{a} \phi=-i e f^{-1} \bar{\phi}\left[-A_{t} \partial_{t}+\left(f A_{R}+O(r)\right) \partial_{R_{*}}+\tilde{O}(r)\right] \phi .
$$

Suppose $A_{a}$ be in the Ashtekar gauge, defined by [28, 36]

$$
\mathcal{L}_{l} A_{\leftarrow} \hat{\leftarrow} \hat{=} 0
$$

where an arrow " $\longleftarrow$ " denotes the pullback to $\mathcal{H}$, e.g. $A_{a}$ denotes the pullback of $A_{a}$ to $\mathcal{H}$. In the Ashtekar gauge, $\Phi_{l}:=-l^{a} A_{a}$ is constant on $\mathcal{H}$, which is the analogue of the static electric potential on the Reissner-Nordström horizon [15]. The potential $A_{a}^{\prime}=A_{a}+(d \alpha)_{a}$ is also in the Ashtekar gauge if and only if

$$
l \cdot d \alpha=D \alpha=\partial_{u} \alpha+U \partial_{r} \alpha+X \partial_{\zeta}+\bar{X} \partial_{\bar{\zeta}} \hat{=} \partial_{u} \alpha+U \partial_{r} \alpha \hat{=} C .
$$

Therefore, for a given $A_{a}$ satisfying (35) with a nonzero $A_{R}$ on $\mathcal{H}$, there exists a gauge transformation satisfying (36) and

$$
\partial_{r} \alpha=-\left(2 A_{R}+U^{-1} C\right)
$$

which makes $A_{a}^{\prime}$ satisfy (35) with

$$
A_{R}^{\prime}=f^{-1} A_{u}^{\prime}+A_{r}^{\prime}=f^{-1}\left(A_{u}+\partial_{u} \alpha\right)+A_{r}+\partial_{r} \alpha \hat{=} A_{R}+\frac{1}{2} \partial_{r} \alpha+f^{-1} C \hat{=} 0 .
$$

Namely, one may always write

$$
-i e \bar{\phi} A_{a} \nabla^{a} \phi=i e f^{-1} \bar{\phi}\left[A_{t} \partial_{t}+\tilde{O}(r)\right] \phi
$$

without loss of generality. The second interaction term is just the complex conjugate of the first one. The last interaction term reads in the above gauge

$$
e^{2}|A|^{2}|\phi|^{2}=e^{2}\left(2 A_{u} A_{r}+\mathcal{A} A_{r}^{2}+2 A_{r} A_{B} \mathcal{B}^{B}+h^{A B} A_{A} A_{B}\right)|\phi|^{2} \hat{=}-e^{2} f^{-1} A_{u}^{2}|\phi|^{2} .
$$

Therefore, under above gauge choice, the interaction part of the action in near horizon region can be written as

$$
\sum_{k} \int d t d R_{*}\left\{i e \bar{\phi}_{k}\left(A_{t} \frac{\partial}{\partial t}\right) \phi_{k}-i e \phi_{k}\left(A_{t} \frac{\partial}{\partial t}\right) \bar{\phi}-e^{2}\left|\phi_{k}\right|^{2} A_{t}^{2}+O(r)\right\}
$$


and the action of the complex scalar field as

$$
S=\sum_{k} \int d t d R_{*} \bar{\phi}_{k}\left[-\left(\frac{\partial}{\partial t}-i e A_{t}\right)\left(\frac{\partial}{\partial t}+i e A_{t}\right)+\frac{\partial^{2}}{\partial R_{*}^{2}}\right] \phi_{k}+O(r) .
$$

It shows that the physics near WIH is an infinite collection of $(1+1)$ dimensional fields in the effective 2-dimensional space-time with a $U(1)$ gauge field.

Again, the boundary condition of neglecting the ingoing modes near WIH is imposed. As reviewed in [32], the consistent form of 2-dimensional Abelian anomaly is

$$
\nabla_{\mu} J^{\mu}=\frac{e^{2}}{4 \pi \sqrt{-g}} \epsilon^{\alpha \beta} \partial_{\alpha} A_{\beta}, \quad \alpha, \beta, \mu=0,1,
$$

where $J^{\mu}$ is the current of the $U(1)$ field. The current is conserved in out region and satisfies above equation in near horizon region. The general solution of Eq.(42) for $J^{\mu}$ is

$$
\begin{aligned}
J_{O}^{R} & =c_{O}, \\
J_{H}^{R} & =c_{H}+\frac{e^{2}}{4 \pi}\left[A_{t}(R)-A_{t}(0)\right],
\end{aligned}
$$

where $c_{O}$ and $c_{H}$ are constants. The vanishing variation of the effective action with respect to the gauge parameter $\Lambda$

$$
\begin{aligned}
0 & =-\delta W=\int d x^{2} \Lambda \nabla_{\mu} J^{\mu} \\
& =\int d x^{2} \Lambda\left[\delta(r-a)\left(J_{O}^{R}-J_{H}^{R}+\frac{e^{2}}{4 \pi} A_{t}\right)+\partial_{R}\left(\frac{e^{2}}{4 \pi} A_{t} \Theta_{-}\right)\right],
\end{aligned}
$$

implies

$$
c_{O}=c_{H}-\left.\frac{e^{2}}{4 \pi} A_{t}\right|_{\mathcal{H}}
$$

Further, the covariant current vanishes at the WIH results in

$$
c_{O}=-\left.\frac{e^{2}}{2 \pi} A_{t}\right|_{\mathcal{H}}=-\frac{e^{2}}{\pi} \Phi_{l}
$$

It is just the charge flux. For Reissner-Nordstöm black hole, as an example, $\Phi_{l}=Q /\left(2 r_{+}\right)$ and Eq.(47) reduces to Eq.(12) in [15]. It is worth to point out that above result is independent on the gauge choice Eq.(35) because of Eq.(42).

\section{GENERALIZATION TO WEAKLY ISOLATED HORIZON IN HIGHER DIMENSIONAL SPACETIME}

The previous discussion can be straightly generalized to the WIH in a higher dimensional spactime. 
Definition 2 (Weakly Isolated Horizon in Higher Dimensional Space-time)

An $(n-1)$-dimensional null hypersurface $\mathcal{H}$ in an $n$-dimensional spacetime $(M, g)$ is said to be a weakly isolated horizon (WIH), if

1) $\mathcal{H}$ has the topology of $\mathbf{K} \times \mathbf{R}$, where $\mathbf{K}$ is an $(n-2)$-dimensional compact Riemannian manifold;

2) the expansion of the null generator of $\mathcal{H}$, whose tangent vector field is $l^{a}$, is zero, i.e. $\Theta_{l}=0$ on $\mathcal{H}$;

3) $T_{a b} v^{b}$ is future causal for any future-directed causal vector $v^{a}$ and $n$-dimensional Einstein equations hold in a neighborhood of $\mathcal{H}$;

4) acting on $l$, Lie derivative $\mathcal{L}_{l}$ and induced covariant derivative $D_{a}$ on $\mathcal{H}$ commute, i.e. $\left[\mathcal{L}_{l}, D_{a}\right] l^{b}=0$ on $\mathcal{H}$.

Again, there exists a 1 -form $\omega_{a}$ such that $D_{a} l^{b} \hat{=} \omega_{a} l^{b}$ by definition.

Now, in the Bondi gauge in a neighborhood of $\mathcal{H}, n$-bein can be expressed in the Bondilike coordinates $\left(u, r, \zeta^{A}\right)$ with $A, B=2, \cdots n-1$ as

$$
\left\{\begin{array}{l}
l^{a}=\partial_{u}+U \partial_{r}+X^{A} \partial_{A} \\
n^{a}=\partial_{r} \\
e^{A a}=\omega^{A} \partial_{r}+\xi^{A B} \partial_{B},
\end{array}\right.
$$

where

$$
U \hat{=} X^{A} \hat{=} \omega_{A} \hat{=} 0
$$

They satisfy the normal condition:

$$
l^{a} l_{a}=0, \quad n^{a} l_{a}=1, \quad n^{a} n_{a}=0, \quad l^{a} e_{a}^{A}=0, \quad n^{a} e_{a}^{A}=0, \quad e^{A a} e_{a}^{B}=\delta^{A B} .
$$

The metric of $n$ dimensional spacetime is 31 ]

$$
\left(g^{\mu \nu}\right)=\left(\begin{array}{ccc}
0 & 1 & 0 \\
1 & 2 U+\delta_{C D} \omega^{C} \omega^{D} & X^{A}+\delta_{C D} \omega^{C} \xi^{D A} \\
0 & X^{B}+\delta_{C D} \omega^{C} \xi^{D B} & \delta_{C D} \xi^{C A} \xi^{D B}
\end{array}\right)
$$

In the Bondi coordinates, $\sqrt{|g|}=\sqrt{h}$ where $\left(h_{A B}\right):=\left(\delta_{C D} \xi^{C A} \xi^{D B}\right)^{-1}$.

The Bondi gauge can be expressed as

$$
\left\{\begin{array}{l}
\nu^{A}:=-n_{\mu ; \nu} e^{A \mu} n^{\nu}=-e^{A \mu} \Delta n_{\mu}=0, \\
\tau^{A}:=l_{\mu ; \nu} e^{A \mu} n^{\nu}=e^{A \mu} \Delta l_{\mu}=0, \\
\kappa^{A}:=l_{\mu ; \nu} e^{A \mu} l^{\nu}=e^{A \mu} D l_{\mu} \hat{=} 0, \\
\gamma:=-l^{\mu} \Delta n_{\mu}=0, \\
\gamma^{[A B]}:=e^{[A|\mu|} \Delta e^{B]}=0, \\
\varepsilon^{[A B]}:=-e^{[A|\mu|} D e_{\mu}^{B]} \hat{=} 0, \\
\alpha^{A}:=n^{\mu} \delta^{A} l_{\mu}=-e^{A \mu} D n_{\mu}=: \pi^{A}, \\
\mu^{[A B]}:=n_{\mu ; \nu} e^{[A|\mu|} e^{B] \nu}=e^{[A|\mu|} \delta^{B]} n_{\mu}=0,
\end{array}\right.
$$

where $D$ and $\Delta$ is defined as before, $\delta^{A}:=e^{A a} \nabla_{a}$. In the Bondi gauge, 1 -form $\omega_{a}$ is expressed as

$$
\omega_{a}=-\varepsilon_{0} n_{a}+\delta_{A B} \pi^{A} e_{a}^{B},
$$


where $\varepsilon_{0}:=n^{\mu} D l_{\mu} \hat{=}$ constant [28]. In addition, the definition of WIH in $n$-dimensional spacetime implies

$$
\rho^{A B}:=e^{A \mu} \delta^{B} l_{\mu} \hat{=} 0
$$

And the angular momentum of WIH in $n$-dimensional spacetime is

$$
J[\varphi]=-\frac{1}{8 \pi} \int_{\mathbf{K}}\left(\varphi^{a} \omega_{a}\right) d V_{n-2},
$$

where $\varphi^{a}$ is a vector field on section $\mathbf{K}$. As before, only $\pi^{A}$ are related to the angular momentum of WIH.

The commutators of the $n$-bein are

$$
\left\{\begin{array}{l}
\Delta D-D \Delta=\gamma D+\varepsilon \Delta+\delta_{A B}\left(\tau^{A}+\pi^{A}\right) \delta^{B} \\
\delta^{A} D-D \delta^{A}=\left(\alpha^{A}-\pi^{A}\right) D+\kappa^{A} \Delta+\frac{\rho}{n-2} \delta^{A}+\delta_{B C}\left(\sigma^{(B A)}+\rho^{[B A]}-\varepsilon^{[B A]}\right) \delta^{C} \\
\delta^{A} \Delta-\Delta \delta^{A}=-\nu^{A} D+\left(\tau^{A}-\alpha^{A}\right) \Delta+\frac{\mu}{n-2} \delta^{A}+\delta_{B C}\left(\lambda^{(B A)}+\mu^{[B A]}-\gamma^{[B A]}\right) \delta^{C} \\
\delta^{A} \delta^{B}-\delta^{B} \delta^{A}=2 \mu^{[A B]} D+2 \rho^{[A B]} \Delta+2 \delta_{C D} \beta^{C[A B]} \delta^{D},
\end{array}\right.
$$

where

$$
\left\{\begin{array}{l}
\sigma^{(A B)}:=\rho^{(A B)}-\frac{\rho}{n-2} \delta^{A B} \quad \text { with } \quad \rho:=\delta_{A B} \rho^{A B} \\
\mu^{A B}:=-e^{A \mu} \delta^{B} n_{\mu} \\
\lambda^{(A B)}:=\mu^{(A B)}-\frac{\mu}{n-2} \delta^{A B} \quad \text { with } \quad \mu:=\delta_{A B} \mu^{A B} \\
\beta^{A B C}:=e^{A \mu} \delta^{B} e_{\mu}^{C} .
\end{array}\right.
$$

They require

$$
\begin{aligned}
& U=\hat{\varepsilon}_{0} r+O\left(r^{2}\right), \quad \kappa^{A}=O(r), \beta^{C A B}=O(1), \quad \lambda^{(A B)}=O(1), \\
& \varepsilon_{0}=\hat{\varepsilon}_{0}+O(r), \quad \partial_{u} \xi^{A B} \hat{=} 0, \pi^{A}=O(r), \quad \omega=O(r) .
\end{aligned}
$$

The d'Alembert operator can be re-written as

$$
\begin{aligned}
= & g^{a b} \nabla_{a} \nabla_{b}=\left(l^{a} n^{b}+n^{a} l^{b}+\delta_{A B} e^{A a} e^{B b}\right) \nabla_{a} \nabla_{b} \\
= & D \Delta+\Delta D+\left(\varepsilon_{0}+\rho\right) \Delta+\mu D-\delta_{A B} \pi^{A} \delta^{B}+\Delta_{\mathbf{K}} \\
= & 2 \partial_{u} \partial_{r}+2 U\left(\partial_{r}\right)^{2}+2 X^{A} \partial_{r} \partial_{A}+\left(\partial_{u} U\right) \partial_{r}+\left(\partial_{r} X^{A}\right) \partial_{A} \\
& +\left(\varepsilon_{0}+\rho\right) \partial_{r}+\mu\left(\partial_{u}+U \partial_{r}+X^{A} \partial_{A}\right)-\delta_{A B} \pi^{A} \delta^{B}+\Delta_{\mathbf{K}}
\end{aligned}
$$

where

$$
\Delta_{\mathbf{K}}=\delta_{A B} \delta^{A} \delta^{B}-\delta_{A B} \delta_{C D} \beta^{C A B} \delta^{D}
$$

is the Laplacian on $\mathbf{K}$. Obviously, it has the same behaviors as in 4-dimensional spacetime when $r \rightarrow 0$. Like the 4-dimension case, the field $\phi$ can also be expressed into a variable separated form $\phi(x)=\sum_{k} \phi_{k}(u, r) F_{k}^{(n-2)}$, where $\left\{F_{k}^{(n-2)}\right\}$ are eigenfunctions of the $(n-2)$ dimensional Laplacian. Introducing tortoise-like coordinates $(t, R)$ as (10), the previous discussions are still valid for the WIH in the higher dimensional spacetime. 


\section{CONCLUSION AND DISCUSSION}

In above work, have shown that the anomaly analysis of Wilczek and his collaborators is indeed applicable to the general weakly isolated horizons. Here, we want to give some remarks on our work.

First, we focus on 4-dim spacetime without cosmological constant in previous work. What we want to emphasis is our derivation also holds for the spacetime with a nonzero cosmological constant. The reason is as follows: Suppose we have a 3-dim null surface $\mathcal{H}$ in a spacetime with nonzero cosmological constant, such that $\mathcal{H}$ satisfies all requirements of definition 1. The Einstein equation with a cosmological constant is

$$
G_{a b}-\Lambda g_{a b}=8 \pi T_{a b}
$$

We can always move the cosmological constant term to the right hand side of the equation and define a new energy-momentum tensor $\tilde{T}_{a b}:=T_{a b}+\Lambda g_{a b} /(8 \pi)$. From the definition of WIH, we have known that $T_{a b}$ satisfies $T_{l l}=0, T_{l m}=T_{l \bar{m}}=0$ (or $T_{l A}=T_{A l}=0$ in higher dimensional spacetime). It is easy to check $\Lambda g_{a b}$ also satisfies these requirements, so the total energy-momentum tensor $\tilde{T}_{a b}$ satisfies the requirements of the definition of WIH. Furthermore, when a positive cosmological constant is present, the spacetime will, in general, have a cosmological horizon, which also satisfies the weakly isolated condition. Therefore, the above derivation also applies to the cosmological horizon. The only difference is that the topology of WIH for negative cosmological constant case is $S \times \mathbf{R}$, where $S$ can be any kinds of 2-dim compact manifold[28].

Second, it is worth to notice that our calculation is just based on Cartan structure equations. This means our calculation can also be used to black holes from other gravitational theories if the black hole horizon is null and has zero expansion, for example the black ring solution, $f(R)$ theory and other theories. This means the Hawking radiation is in fact a kinematical effect of spacetime [37, 38, 39, 40, 41].

\section{Acknowledgments}

We would like to thank Prof. Z. Chang and Prof. H.-Y. Guo for helpful discussion. The project is partly supported by the Natural Science Foundation of China under Grant Nos. 90403023, 10605006, 10705048, 10775140, 10731080 and Knowledge Innovation Funds of CAS (KJCX3-SYW-S03).

[1] S. Hawking, Nature (London) 248 (1974) 30.

[2] S. Hawking, Commun. Math. Phys. 43 (1975) 199.

[3] W. G. Unruh, Phy. Rev. D 10 (1974) 3194.

[4] J. B. Hartle and S. W. Hawking, Phys. Rev. D 13 (1976) 2188.

[5] T. Damoar and R. Ruffini, Phys. Rev. D 14 (1976) 332.

[6] G. W. Gibbons and S. W. Hawking, Phys.Rev.D 15 (1977) 2752.

[7] G. W. Gibbons and M. J. Perry, Proc. R. Soc. Lond. A 358 (1978) 467.

[8] M. K. Parikh and F. Wilczek, Phys. Rev. Lett. 85 (2000) 5042. 
[9] S. Shankaranarayanan, T. Padmanabhan and K. Srinivasan, Class. Quant. Grav. 19 (2002) 2671.

[10] A. J. M. Medved and Elias C. Vagenas, Mod. Phys. Lett. A 20 (2005) 1723.

[11] R. Balbinot, Phys. Rev. D 33 (1986) 1611.

[12] Z. Zhao and X. X. Dai, Chin. Phys. Lett. 10 (1991) 548.

[13] J. Y. Zhu, J. Zhang and Z. Zhao, Inter. J. Theor. Phys. 33 (1994) 2049.

[14] S. P. Robinson and F. Wilczek, Phys. Rev. Lett. 95 (2005) 011303.

[15] S. Iso, H. Umetsu and F. Wilczek, Phys. Rev. Lett. 96 (2006) 151302.

[16] S. M. Christensen and S. A. Fulling, Phys. Rev. D 15 (1977) 2088.

[17] K. Murata and J. Soda, Phys. Rev. D 74 (2006) 044018.

[18] S. Iso, H. Umetsu and F. Wilczek, Phys. Rev. D 74 (2006) 044017.

[19] E. C. Vagenas and S. Das, JHEP 0610 (2006) 025.

[20] S. Iso, T. Morita and H. Umetsu, "Quantum Anomalies at Horizon and Hawking Radiations in Myers-Perry Black Holes", arXiv:hep-th/0612286.

[21] M. R. Setare, Eur. Phys. J. C 49 (2007) 865-868.

[22] Q. Q. Jiang, S. Q. Wu and X. Cai, Phys. Rev. D75 (2007) 064029.

[23] K. Xiao, W.-B. Liu, H.-B. Zhang, Phys. Lett. B 647 (2007) 482-485.

[24] Z. Xu and B. Chen, Phys. Rev. D 75 (2007) 024041.

[25] S. Das, S. P. Robinson and E. C. Vagenas, "Gravitational anomalies: A Recipe for Hawking radiation", arXiv:0705.2233.

[26] B. Chen and W. He, "Hawking Radiation of Black Rings from Anomalies", arXiv:0705.2984.

[27] C.-G. Huang, J.-R. Sun, X. Wu and H.-Q. Zhang, "Gravitational Anomaly and Hawking Radiation of Brane World Black Holes", arXiv:0710.4766.

[28] A. Ashtekar and B. Krishnan, Living Rev. Rel. 7 (2004) 10.

[29] X. Wu and S. Gao, Phys. Rev. D 75 (2007) 044027.

[30] R. Penrose and W. Rindler, Spinors and spacetime Vol.1, Cambridge University Press, Cambridge, 1984.

[31] E. T. Newman and K. P. Tod, "Asymptotically flat space-time", in General Relativity and Gravitation, Vol.2, ed. by A. Held, New York, Plenum, 1980.

[32] R. Bertlmann, Anomalies In Quantum Field Theory, Oxford Science Publications, Oxford, 2000.

[33] L. Alvarez-Gaume and E. Witten, Nulc. Phys. B 234 (1984) 269.

[34] W. A. Bardeen and B. Zumino, Nulc. Phys. B 244 (1984) 421.

[35] R. Bertlamnn and E. Kohlprath, Ann. Phys. (N.Y.) 288 (2001) 137.

[36] A. Ashtekar, S. Fairhurst and B. Krishnan, Phys. Rev. D 62 (2000) 104025.

[37] W. G. Unruh, "Experimental black-hole evaporation?", Phys. Rev. Lett. 46, 1351 (1981).

[38] M. Visser, "Acoustic black holes: Horizons, ergospheres, and Hawking radiation", Class. Quant. Grav. 15, 1767 (1998).

[39] M. Visser, "Hawking radiation without black hole entropy", Phys. Rev. Lett. 80, 3436 (1998).

[40] C.-G. Huang and H.-Y. Guo, "A new kind of uniformly accelerated reference frame", Int. J. Mod. Phys. 15, 1035 (2006).

[41] C.-G. Huang and J.-R. Sun, "Thermodynamic Properties of Spherically-Symmetric, Uniformly-Accelerated Reference Frames", gr-qc/0701078, to appear in Commun. Theor. Phys.. 